\title{
Antecedentes, realidades y retos de los programas nacionales para la Reforma Rural Integral
}

\section{Background, realities and challenges of the national programs for the Comprehensive Rural Reform}

Yady Nohelia Figueroa-Torres ${ }^{1}$ Sandra Milena Pérez-Bello ${ }^{2}$

Fecha de recepción: 22 de junio de 2017

Fecha de aprobación: 3 de noviembre de 2017

Claudia Liliana Villamarín-Monroy ${ }^{3}$

Alfonso Rincón-Pérez ${ }^{4}$

DOI: https://doi.org/10.19053/01228420.v15.n1.2018.7761

\section{Resumen}

Los acuerdos entre las Fuerzas Armadas Revolucionarias de Colombia -FARC- y el gobierno nacional, en La Habana, incluyen un punto de gran relevancia para el sector rural: la Reforma Rural Integral; esta contempla no solo el tema productivo, sino también el concepto de territorio y todos los actores inmersos en él, y busca mejorar las condiciones de vida de la población rural a través de una transformación estructural que respete las comunidades y sus identidades y brinde oportunidades para promover el Desarrollo Rural.

Este artículo busca analizar el tercer aspecto del punto uno del acuerdo, los planes nacionales para la reforma rural integral, cuyo principal objetivo es la superación de la pobreza y el cierre de la brecha campo-ciudad. Para el artículo, en primer lugar, se realizó una descripción de la situación de la pobreza rural en Colombia y de la situación de las brechas campo-ciudad, y, en segundo lugar, se revisaron los aspectos que plantea la Reforma Rural Integral para superar la pobreza y disminuir estas brechas; esto con el fin de conocer en qué contexto se plantea implementar esta reforma.

Palabras clave: acuerdo de paz; FARC; Pobreza rural; Reforma Rural Integral.

1 Universidad Pedagógica y Tecnológica de Colombia (Tunja-Boyacá, Colombia).

2 Universidad Nacional de Colombia (Bogotá-Distrito Capital, Colombia)

3 Universidad Pedagógica y Tecnológica de Colombia (Tunja-Boyacá, Colombia). claudia.villamarin@uptc.edu.co.

4 Universidad Pedagógica y Tecnológica de Colombia (Tunja-Boyacá, Colombia). 


\begin{abstract}
The peace agreements between the Colombian Revolutionary Armed Forces -FARC- and the National Government in Havana include a point of great relevance for the rural sector: the Comprehensive Rural Reform. This reform contemplates not only the productive aspect but also the concept of territory and all the actors involved in it; additionally, it seeks to improve the living conditions of the rural population through a structural transformation that respects the communities and their identities, and that provides opportunities to promote Rural Development.

This article seeks to analyze the third aspect of point one of the agreement, the national plans for the integral rural reform, whose main objective is to overcome poverty and close the rural-city gap. In order to achieve this, first, the situations of rural poverty and rural-city gap in Colombia were described, and second, the proposals of the comprehensive rural reform to overcome poverty and reduce these gaps were revised, with the aim of understanding the context in which this reform is proposed to be implemented.
\end{abstract}

Keywords: agreement, Comprehensive Rural Reform, land; poverty.

\title{
Para citar este artículo:
}

Figueroa-Torres YN., Pérez-Bello SM., Villamarín-Monroy CL., Rincón-Pérez A. Antecedentes, realidades y retos de los programas nacionales para la Reforma Rural Integral. Ciencia y Agricultura. 2018; 15(1): 101-110. 


\section{Introducción}

Colombia finaliza el año 2016 con un hecho tanto histórico como controversial: la firma de los acuerdos de paz entre el Gobierno y las FARC. Se han escuchado las versiones tanto de opositores como defensores al respecto que enfatizan en uno $u$ otro aspecto; pero lo que es indudable es que este acuerdo va a marcar un hito en la transformación estructural del campo y en las políticas del sector rural, porque es ahí donde comenzó el conflicto armado.

En el acuerdo se le da al sector rural una importancia fundamental, tanto que el primer punto acordado tiene como objetivo la transformación estructural del campo, con el fin de impulsar el desarrollo del país, lograr la integración de las regiones, garantizar la seguridad alimentaria, lograr el reconocimiento e inclusión del campesinado, poner fin a la concentración en la propiedad de la tierra y el retraso rural (1), con el objetivo de lograr una paz estable y duradera. Para lograr esta transformación, el punto uno del acuerdo (Reforma Rural Integral -RRI-) plantea tres aspectos: el primero hace referencia al acceso y uso de la tierra; el segundo, a los programas de desarrollo con enfoque territorial, y el tercero, a los planes nacionales para la reforma rural integral.

En el presente artículo se analiza el tercer aspecto del punto uno del acuerdo (planes nacionales para la Reforma Rural Integral), cuyo principal objetivo es la superación de la pobreza y el cierre de la brecha campo-ciudad. Para realizar este análisis se consideró necesario, en primer lugar, conocer la situación de la pobreza rural en Colombia y la situación de las brechas campo-ciudad, y, en segundo lugar, revisar los aspectos que plantea la Reforma Rural Integral para superar la pobreza y disminuir estas brechas; esto con el fin de conocer en qué contexto se plantea implementar esta reforma.

\section{La pobreza en Colombia}

El enfoque de capacidades y derechos, liderado por Amartya Sen, define la pobreza, no como la carencia de bienes ni de la utilidad que pueda tener una persona en particular, sino como la inca- pacidad y la falta de libertad de elección para poder realizar determinadas acciones que permitan el ser y el hacer, lo cual se traduce en el bienestar; esta perspectiva alimenta, en buena medida, el enfoque de Desarrollo Humano que promueve la Organización de Naciones Unidas (ONU), el cual pone a las personas como la razón de ser de dicho desarrollo (2).

En Colombia, la reducción de la pobreza ha sido considerada en todos los planes de gobierno, buscando alternativas para afrontarla, y existe una amplia oferta de instituciones (3) y programas a nivel nacional que apuntan a este objetivo.

Se tienen tres indicadores para calcular la pobreza: Necesidades Básicas Insatisfechas (NBI), Índice de Pobreza Multidimensional (IPM) y Pobreza Monetaria (2), los cuales, antes de ser excluyentes, son complementarios entre sí.

EI NBI busca establecer las carencias que tienen los hogares, de acuerdo con un umbral establecido, con unas necesidades básicas definidas en cinco dimensiones (1) Educación, el analfabetismo y el bajo logro educativo. 2) Niñez y juventud, que comprende trabajo infantil, acceso a servicios de cuidado de la primera infancia, así como inasistencia y rezago escolar. 3) Trabajo, que tiene en cuenta el desempleo de larga duración y la tasa de informalidad. 4) Salud, en cuanto al acceso y aseguramiento. 5) Vivienda y servicios públicos, que agrupa las variables de acceso a fuentes de agua mejoradas, eliminación de excretas, hacinamiento crítico y pisos o paredes inadecuados (2): viviendas inadecuadas, viviendas con hacinamiento crítico, viviendas con servicios inadecuados, viviendas con alta dependencia económica y viviendas con niños en edad escolar que no asisten a la escuela (3) indican necesidades básicas insatisfechas, y se considera pobre un hogar que presenta al menos una de las anteriores privaciones, mientras que un hogar se encuentra en situación de miseria si incumple con dos o más de los criterios anteriormente descritos (2).

En la Gráfica 1 se observa que, en Colombia, en el período 1970-2010, el porcentaje de personas con al menos una NBI ha disminuido considerablemen- 
te, debido principalmente a la inversión social destinada a mejorar las características de la vivienda, el hacinamiento, la cobertura en servicios básicos, la escolaridad y la tasa de dependencia. Durante el período 1973-1993, este indicador se redujo de $58.9 \%$ al $37.2 \%$ en las zonas urbanas, y en las zonas rurales disminuyó del $87.9 \%$ al $58.9 \%$, observándose mayor orientación del gasto público y de servicios del Estado hacia las zonas urbanas del país. De 1997 al 2009 hubo una reducción de 8.3 puntos, ubicándose el indicador en 17.6; en este periodo, todos los factores constitutivos del índice, a excepción del correspondiente a vivienda, que permanece constante, contribuyeron a la reducción de la pobreza (3).

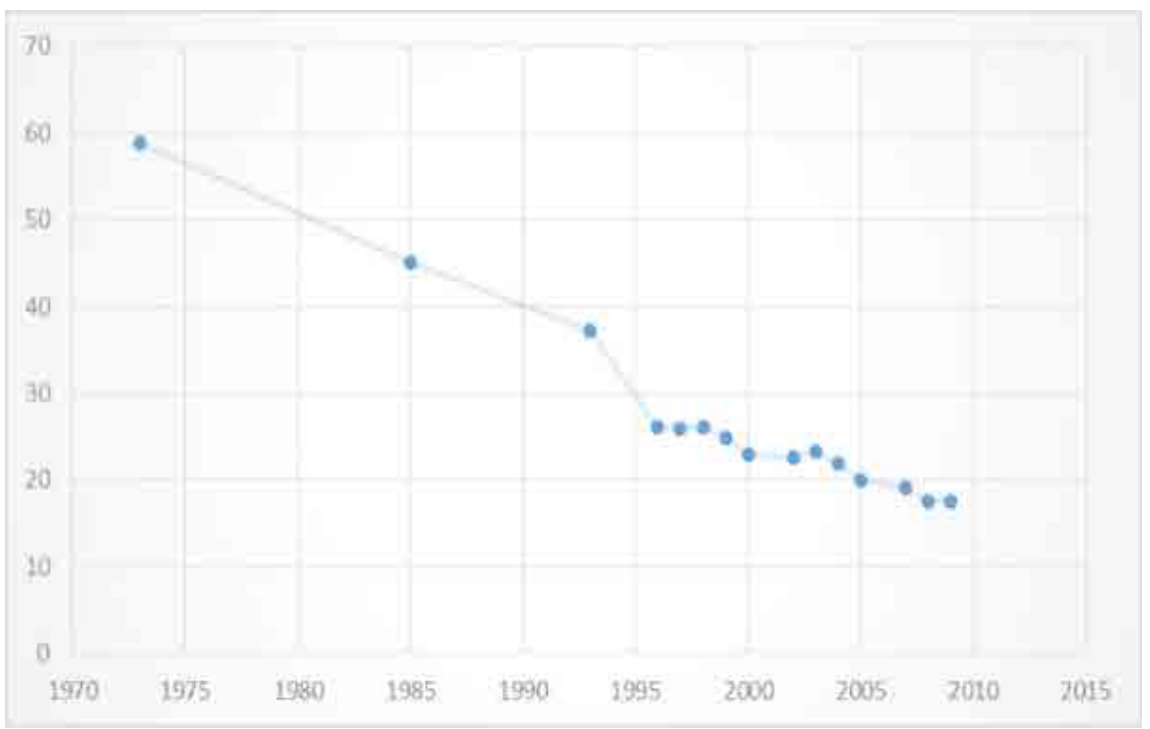

Gráfica 1. Porcentaje de la población con necesidades básicas insatisfechas en Colombia durante el periodo 1970-2010. Fuente: DANE.

El Índice de Pobreza Multidimensional mide la incidencia, la brecha y la severidad de la pobreza, bajo cinco dimensiones, que trascienden la variable de ingreso y el enfoque de carencias. Como lo indica la Gráfica 2, a nivel nacional el indicador se mantuvo constante durante el período 1991-1996; sin embargo, para el sector rural aumentó en casi 10 puntos, pasando del 68.4 al 77.4. A partir del año 2000 el indicador, que a nivel nacional se ubicaba en 59.8, y a nivel rural, en 82.6, comienza a disminuir de manera constante hasta el 2014, cuando se ubica en 21.9 y 44.1 , respectivamente. 


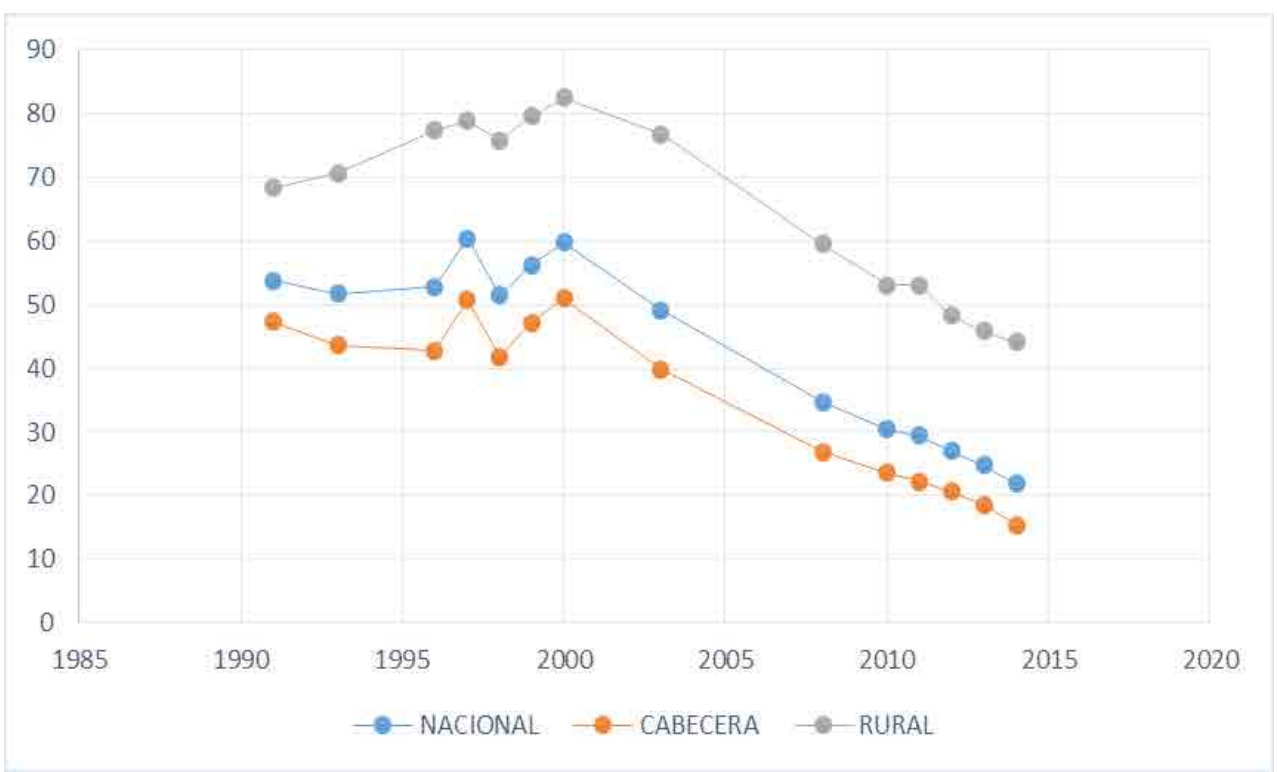

Gráfica 2. Índice de Pobreza Multidimensional en Colombia durante el período 1990-2014. Fuente: DANE.

Aunque la pobreza se ha reducido en las zonas rurales, la brecha entre la población urbana y la rural ha aumentado 7.6 puntos entre 1991-2014; en 1991 era de 21 puntos, y en 2014, de 28.7 (3). A pesar de los esfuerzos fiscales que se han realizado para mejorar las condiciones de vida en el sector rural con la provisión de bienes y servicios sociales, estos por sí solos no han sido suficientes para lograr una reducción sostenida de la pobreza y mayor equidad.

El indicador Pobreza Monetaria calcula los niveles de ingreso mínimo para adquirir una canasta de bienes alimentarios y no alimentarios que permita tener un adecuado nivel de vida, definiendo así la
Línea de Pobreza (LP); en tanto que la Línea de Indigencia, o pobreza extrema, está dada por el ingreso mínimo para adquirir una canasta de bienes exclusivamente alimentarios que cumpla con los requerimientos nutricionales.

Como se puede observar en la Gráfica 3, la pobreza monetaria disminuyó levemente a nivel nacional hasta 1996, ubicándose en $50.8 \%$; en el período de 1996 a 1999, el indicador nacional subió a $57.4 \%$, y en el sector rural, a $78.1 \%$, debido principalmente a la crisis económica de finales de 1990, el desempleo y la caída de los ingresos rurales (4). 


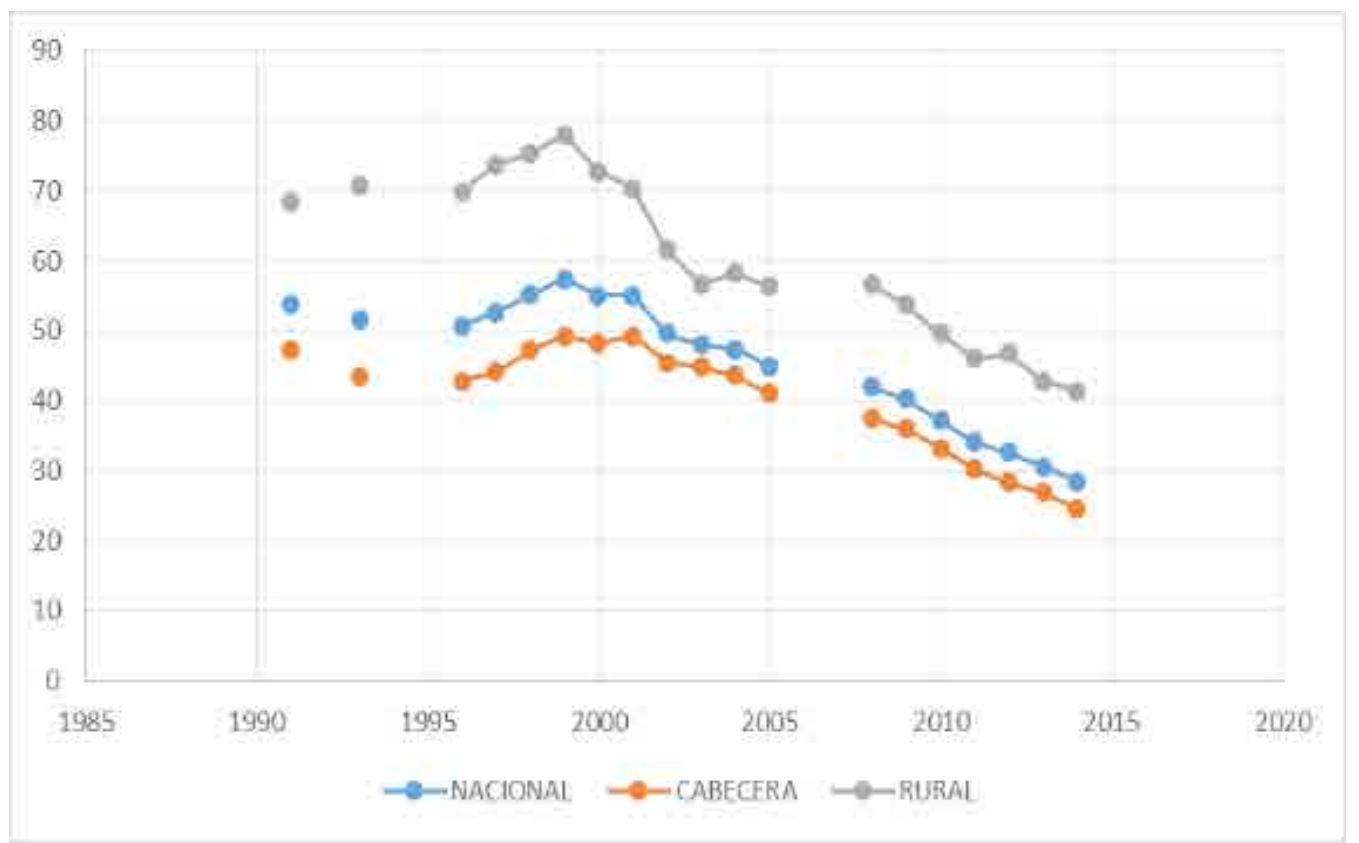

Gráfica 3. Indicador de Pobreza Monetaria en Colombia en el período 1990-2014. Fuente: DANE.

A partir del año 2000, los niveles de pobreza monetaria han tendido a la baja, gracias a la recuperación importante de los índices de empleo, los ingresos y un mayor crecimiento económico.

A pesar de que la pobreza ha disminuido en el período de análisis, Colombia enfrenta una grave situación en materia de desigualdad y brechas; el coeficiente de Gini para el país, en relación con los ingresos, se mantuvo en 0,538 en 2014, un valor similar al obtenido en 2012 y $2013(0,539)$, y apenas 0,02 puntos por debajo del coeficiente registrado una década atrás, posicionando a Colombia como el tercer país más desigual de América Latina.

Como se plantea en el artículo Pobreza, brechas y ruralidad en Colombia (5), se requieren dos estrategias fundamentales para superar la situación de la pobreza rural; primera, una redistribución de la tierra, y segunda, mejorar los medios de vida en las zonas rurales, a través de políticas sociales activas con mayores inversiones públicas en educación, salud e infraestructura, aspectos en los cuales se fundamente el acuerdo de La Habana y la reforma rural integral.

\section{Redistribución de la tierra}

El Centro Nacional de Memoria Histórica, en su libro La política de reforma agraria y tierras en Colombia. Esbozo de una memoria institucional, de 2013 (6), relata cómo han sido los intentos de reforma agraria desde 1960, y frente a lo cual, Absalón Machado, uno de los más expertos en temas de agricultura manifiesta: "vivimos repitiendo los errores del pasado por no conocer lo que se ha hecho en las políticas públicas" (7). El autor hace referencia a un claro desamparo en el contexto de las políticas agrarias, lo que lleva a que se creen políticas sin viabilidad y fracasen; esto ocurre cuando el Estado genera políticas en el contexto de períodos de gobierno y no se tienen en cuenta las experiencias de los gobiernos anteriores, se pierde la visión hacia el futuro, puesto que las reformas agrarias tienden a ser desarrolladas en períodos de tiempo de menos de diez años; por otra parte, el sector privado, con el fin de defender sus intereses, se opone al cambio.

Machado, en su libro La reforma rural: una deuda social y política (8), afirma que en América Latina, para resolver los problemas estructurales en las décadas de los sesenta y setenta se tenía como ob- 
jetivo principal la redistribución, donde el Estado intervenía directamente, se denominó en ese entonces "Reforma Agraria Histórica"; era una época de intervencionismo y centralismo estatal, donde se pensaba que si el Estado no impulsaba los cambios agrarios ninguna otra entidad lo podría lograr.

Una de las razones por las que en los años ochenta las reformas agrarias quedaron relegadas fue porque se dio prioridad a la búsqueda de equilibrios macroeconómicos y a las políticas que abrieran la economía y generaran crecimientos más dinámicos. Todo esto ha llevado a que el problema agrario en Colombia no se haya solucionado; como lo manifiesta Rudolf Hommes (9), es evidente el poco interés del Estado en mejorar las condiciones de vida de los campesinos, y eso ha hecho que el sector agropecuario tenga bajos índices de productividad y altos índices de violencia.

El planteamiento de la redistribución de la tierra se vuelve a retomar con el acuerdo de paz, que toma elementos orientados a resolver los problemas agrarios para garantizar un mayor acceso y uso de tierras improductivas, formalización de la propiedad, manejo de la frontera agrícola y protección de las zonas de reserva.

Pero si bien se pudiera realizar esta reforma en la tenencia de la tierra, por sí sola no va a garantizar la superación de la pobreza; se hace necesario implementar la reforma rural integral para mejorar los ingresos de los habitantes del sector y garantizar un acceso adecuado a servicios y bienes públicos y así superar la brecha urbano-rural.

\section{Reforma Rural Integral}

El acuerdo de La Habana plantea tres líneas con las cuales van a lograr el objetivo de superar la pobreza y disminuir las brechas campo-ciudad, demandas que han sido reconocidas no solo en este documento, sino por estudios anteriores como el realizado por la Comisión Económica para América Latina y el Caribe (CEPAL): "Las brechas entre el campo y la ciudad en Colombia 1990-2003 y propuestas para reducirlas" (10), donde se plantea que se debe dotar al campo de medios de trabajo y capacidades para generar mayor desarrollo rural; esto se debe realizar a través de un mayor acceso a servicios básicos sociales (acueducto, energía, teléfono, salud y educación), de la redistribución de tierras según su uso productivo, de consolidar la agricultura familiar y fortalecer la actividad agropecuaria, con infraestructura vial, riego, investigación, acceso a créditos, incentivos e impulso a las cadenas de las alianzas productivas, tanto en las actividades agropecuarias como no agropecuarias y microempresariales.

Así mismo, el tema de disminución de las brechas campo-ciudad ha sido analizado en los planes de gobierno de los presidentes Samper, Pastrana, Uribe y Santos, cuyas estrategias iban dirigidas a elevar la competitividad de la producción agropecuaria, priorizando zonas con ventajas comparativas y cadenas productivas enfocadas al mercado internacional.

Cabe aclarar que aunque los planes de gobierno anteriores plantean esta temática, el acuerdo de La Habana profundiza estrategias en las que se le da gran relevancia al territorio y sus comunidades, haciéndolos partícipes del diseño y ejecución de los programas para superar la pobreza y lograr mayor equidad entre el campo y la ciudad.

Por otra parte, el acuerdo maneja ciertos principios que le dan una connotación social e incluyente, en donde se considera el territorio no solo como los terrenos de la nación, sino como una articulación entorno, recursos naturales y actores sociales en determinada área con particulares características que lo definen.

Entre otros, uno de los principios fundamentales en que se enmarca esta reforma es el de la igualdad y el enfoque de género, que, infortunadamente, algunos malinterpretaron dándole una connotación de orientación sexual, pero realmente es darle mayor participación, protección y oportunidades a la mujer rural, vista como una gran protagonista del desarrollo rural colombiano que merece acceso a todo lo que le beneficie en su territorio.

Otro principio que hace parte de la innovación que ofrece esta reforma es el bienestar y buen vivir, como objetivo final para erradicar la pobreza 
y mejorar las condiciones y calidad de vida rural, respetando siempre los territorios, las etnias y las tradiciones culturales y reconociendo y diferenciando campesinos y demás etnias presentes en el campo.

Marca un punto álgido, un tercer principio que se ha considerado y que ha sido dejado de lado en todos los planes de gobierno: priorizar territorios aquejados por el conflicto, ya que se han priorizado territorios con ventajas comparativas para la producción dirigida al mercado internacional. El acuerdo pretende, con la reforma rural integral, que el expedito actuar del gobierno se dé en función de los lugares más golpeados por el conflicto, la pobreza y el abandono institucional y dando lugar al afán por proteger y reparar de alguna manera a las víctimas que dejó día tras día el conflicto.

Para implementar la reforma rural integral, la primera línea planteada es la infraestructura y adecuación de vías, para lograr el acceso a servicios sociales y de mercados, asegurar el derecho a la alimentación y mejorar los ingresos de las familias campesinas. Con esta estrategia se plantea mejorar la infraestructura en vías terciarias y el riego, con el objetivo de "impulsar la producción familiar y la economía campesina", mejorar la infraestructura eléctrica y de conectividad, con el fin de mejorar la calidad de vida en el campo; todo esto con un plus que radica en mayor participación de la comunidad campesina, mayor asistencia técnica, estímulo a la economía e introducción de tecnología.

La importancia del mejoramiento de vías terciarias en el país lo explica un informe de Fedesarrollo en 2014 (12), donde indica que ante una deficiente infraestructura se presenta una baja productividad, y en este contexto es evidente que una mejora en las vías que conecten las áreas rurales con las cabeceras urbanas reduciría los índices de pobreza y aumentaría el desarrollo regional. Fedesarrollo (12) estima que cerca de $136.000 \mathrm{~km}$ de vías terciarias se encuentran en pésimas condiciones; las densidades más altas de este tipo de vías se concentran en el centro del país y en algunos municipios del Caribe, en cercanías a las grandes ciudades, lo cual evidencia las disparidades regionales.
Por otra parte, en lo referente a la infraestructura de riego, el Instituto Colombiano de Desarrollo Rural INCODER-, en el informe Análisis, Diseño y Construcción de Distrito de Riego del 2015, explica cómo la agricultura consume cerca del $54 \%$ del agua dulce, lo que convierte los sistemas de riego en un importante elemento de desarrollo agrícola; pero a pesar de esto, de los 6.6 de millones de hectáreas de superficie cultivable, solo el $14,3 \%$ cuenta con sistemas de riego, lo que implica grandes atrasos en el campo colombiano.

La segunda línea planteada en el acuerdo de La Habana es el desarrollo social, que incluye un plan nacional de salud rural, un sistema de educación adecuado, acceso a vivienda y agua potable, con el fin de garantizar condiciones de vida dignas en el sector rural.

El sistema de salud en Colombia es de competencia, con una mezcla de público, privado y mercados, regulada por la ley 100 de 1993, con la cual la distribución de recursos pasa a entes territoriales. En 1993, el $23.7 \%$ de la población se encontraba afiliada al sistema general de salud, y en 2009 se encontraba afiliado el $93.4 \%$; este aumento se dio principalmente en los años 1997-1999, por aumento en el régimen contributivo, y a partir del 2003, por aumento en el régimen subsidiado (13).

La salud, aunque ha logrado alcanzar la universalidad, ha tenido inconvenientes en acceso a los servicios y equidad, como lo manifiesta la Cruz Roja (14); en zonas rurales la población presenta mayores dificultades de acceso a servicios de salud, por lo cual se requiere un modelo diferenciado que tenga en cuenta que la población rural es dispersa y tiene características geográficas y demográficas diferentes a las de las comunidades concentradas en centros urbanos, $y$ esto es considerado uno de los grandes desafíos que debe afrontar el Estado colombiano.

En relación con la educación, ha sido considerada uno de los pilares en los que los planes de gobierno fundamentan el aumento de productividad y competitividad para el sector, y como el motor de una concepción de desarrollo sostenible a escala humana; sin embargo, los años de educación alcanzada por la población colombiana son aún muy bajos (15). A nivel 
nacional, el analfabetismo de la población mayor de 15 años disminuyó de $29 \%$, en 1964, a $12 \%$, en 1985 , y para inicios de la década de los noventa, a $3.4 \%$; en el 2002 se redujo a $2.55 \%$, y en el 2012, a $1.66 \%$, mientras que en la población rural se alcanza un $12,5 \%$, cifra alta comparada con el promedio nacional (16).

Adicionalmente, al analizar los años de educación entre las zonas rural y urbana se observa que a inicios de la década de los noventa la población urbana tenía 7,4 años promedio de educación, y la rural, 3,8 años; en el 2012 el promedio era de 9.5 años a nivel urbano, mientras que en las zonas rurales era de 7,8 años (16). El bajo promedio escolar en las zonas rurales es consecuencia de la falta de ingresos de las familias; especialmente en educación media la inasistencia de los jóvenes es muy alta, porque pasan a ser parte de la fuerza laboral; así mismo, factores de orden público y pertinencia de la educación impactan negativamente la cobertura y calidad, lo cual se convierte en una trampa de la pobreza.

Por otra parte, a inicios de 1990 una cuarta parte de las viviendas del sector rural no eran adecuadas; para el año 2000 este porcentaje se había disminuido al $14,8 \%$, gracias en parte a los subsidios entregados por el Estado. El déficit habitacional rural, medido como el número de hogares que carecen de vivienda propia, es del $34 \%$ (15).

La tercera línea planteada en el acuerdo de La Habana para lograr reducir la pobreza y disminuir las brechas campo-ciudad son los estímulos a la producción agropecuaria, los cuales están basados en fomentar la economía solidaria para el fortalecimiento de las capacidades de los productores a través de asistencia técnica, acceso a bienes y servicios sociales, crédito, mercadeo y formalización laboral, con el fin de garantizar el derecho a la alimentación de todos los colombianos. En este marco, las empresas de economía solidaria han sido una estrategia de los diferentes planes de gobierno para fomentar alianzas productivas y la consolidación de agronegocios entre pequeños productores e industriales, pero no han tenido el impacto esperado en la generación de ingresos para los productores.
Por otra parte, la asistencia técnica se ha desmejorado en los últimos años, especialmente para los pequeños productores. La Encuesta Nacional Agropecuaria indica que solo el $8 \%$ de las fincas han usado este servicio y sus dueños se sienten insatisfechos con él, por lo cual deberá diseñarse de tal manera que permita fortalecer las capacidades productivas. Los problemas del sistema actual son varios: la cobertura sigue siendo inadecuada, la calidad no es buena, la institucionalidad dificulta la coordinación, el enfoque sigue siendo lineal y el financiamiento es poco apropiado; la asignación de recursos se da a través de convocatorias, que son excluyentes y no permiten planear a mediano o largo plazo (17).

En lo concerniente al crédito, según el Censo Agropecuario 2014, solo el $11 \%$ de los productores solicitaron un crédito el año anterior; entre las principales razones de los productores para no acudir al crédito formal, según la Encuesta Longitudinal Colombiana de la Universidad de los Andes-ELCA, están el temor de incumplir con los pagos o de perder la garantía (23\%), no tener bienes para dar en garantía (9.9\%) y los requisitos y papeleos exigidos con un costo del crédito del $26.4 \%$ (18).

El tema de la comercialización se ha convertido en una de las grandes debilidades de la agricultura colombiana, dado que se presentan altos índices de intermediación y altos costos de transporte, lo que le resta competitividad a los productos. Los principales cuellos de botella del sistema actual son la falta de una adecuada institucionalidad, infraestructura deficiente, falta de adopción de estándares de calidad y de buenas prácticas, el abuso de posición dominante en ciertos eslabones de las cadenas y la falta de información (17).

\section{Conclusiones}

La Reforma Rural Integral -RRI- planteada en los acuerdos de La Habana retomó temas trascendentales para el sector agropecuario colombiano, como la redistribución de la tierra y la reforma estructural del campo, y con estos propósitos plantea políticas para el sector a largo plazo y articuladas con todos los actores del territorio; reconoce a campesinos, indígenas, raizales y afros, entre otros, como parte de los territorios, y está diseñada para que estas políticas 
no solo respondan a intereses de particulares, sino para el bienestar general de la población del área rural. También procura dar un enfoque territorial, de manera que el desarrollo rural se dé en cada lugar, teniendo en cuenta potencialidades y fortalezas, al igual que debilidades y necesidades; adicionalmente, busca involucrar a las comunidades para que ayuden a construir y diseñar los programas que más favorezcan el desarrollo de estos sitios que han sido tan duramente golpeados por el conflicto.

Igualmente, el acuerdo busca que la calidad de vida en estos territorios mejore notablemente, fortaleciendo la institucionalidad para reducir la brecha campo-ciudad, brindando facilidades de acceso a salud, educación, vivienda rural y legalización de predios; mejorando en infraestructura y protegiendo y garantizando la producción y comercialización de los productos agropecuarios, al igual que toda actividad que se desarrolle a nivel rural.

El principal reto en este momento es que lo planteado en el acuerdo de La Habana se implemente de manera coordinada, y que se garanticen esquemas de acceso efectivo a la población rural y una mayor generación de ingresos, para así superar la pobreza y la brecha campo-ciudad.

Por otra parte, es fundamental que todos los colombianos comprendan que con los acuerdos de La Habana están reconociendo la importancia del campo y el alto grado de abandono que ha tenido del Estado, siendo esta una de las causas del proceso de violencia y que ha sumido al sector en un círculo vicioso en torno a la pobreza.

Por último, para que una política pública dirigida al sector agrario sea exitosa, cada gobierno debe tener en cuenta las experiencias, claro está, pensando en que estas políticas sean aplicables aún después de 10 años. Un común denominador de los textos consultados en las diferentes épocas es que existía la mentalidad de que jamás se podría lograr una reforma agraria que beneficiaría a los agricultores y esto con el acuerdo de La Habana, puede cambiar.

\section{Referencias}

(1) Acuerdo final para la terminación del conflicto y la construcción de una paz estable y duradera. Gobierno nacional y delegados FARC. Habana - Cuba. 2016.
(2) Herrera F. Objetivos de desarrollo sostenible. Colombia. Herramienta de aproximación al contexto local. PNUD, Programa de las Naciones Unidas para el Desarrollo. 2015.

(3) DNP. Bases del Plan Nacional de Desarrollo 2014-2018. "Todos por un nuevo país". Imprenta Nacional, 2014.

(4) DNP. Plan Nacional de Desarrollo 2002-2006. "Hacia un estado comunitario". Imprenta Nacional, 2002.

(5) Parra RI., Ordóñez LA., Acosta CA. Pobreza, brechas y ruralidad en Colombia. Coyuntura Económica, 2013; 15-36.

(6) Centro Nacional de Memoria Histórica. La política de reforma agraria y tierras en Colombia. 2013.

(7) La verdad Abierta. Las reformas rurales en los últimos 50 años. Recuperado de: http://www.verdadabierta.com/tierras/investigaciones/5045

(8) Machado, A. La reforma rural: una deuda social y política. 2009.

(9) Hommes R. La productividad de los campesinos. El Tiempo, 17 de enero 2013.

(10) Forero-Álvarez J., Ezpeleta Merchán S. Las brechas entre el campo y la ciudad en Colombia 1990-2003 y propuestas para reducirlas. Serie Estudios y Perspectivas. Agosto de 2007.

(11) Villar L., Ramírez JM. Infraestructura regional y pobreza rural. Fedesarrollo, Documento de trabajo N. ${ }^{\circ}$ 61. 2014.

(12) Ministerio de Protección Social de Colombia. Recuperado de: http//www.prevencionintegral.com. 2005.

(13) Cruz Roja Colombiana. La salud rural en Colombia, un modelo diferenciado. El Tiempo, 18 de agosto de 2016.

(14) DNP. Plan Nacional de Desarrollo 2006-2010, "Estado comunitario, desarrollo para todos". Imprenta Nacional, 2007.

(15) PNUD. Objetivos de desarrollo del milenio 2014. PNUD. 2015.

(16) DNP. Misión para la transformación del campo - diagnóstico. Imprenta Nacional, 2015.

(17) Asobancaria. Una mirada al crédito agropecuario. Semana Económica, noviembre de 2014.

(18) OECD. Revisión de la OCDE de las Políticas Agrícolas: Evaluación y Recomendaciones de Política. 2015.

(19) Pardo Martínez LP., Huertas de Mora MV. Tendencias en la gestión de empresas sin ánimo de lucro. Caso: cooperativas. Sector cooperativo y sistema solidario en Colombia. Universidad Cooperativa de Colombia, 2014.

(20) DNP. Misión para la transformación del campo, sistema nacional de crédito agropecuario, propuesta de reforma. 2014.

(21) DNP. Plan Nacional de Desarrollo 1998-2002 "Cambio para construir la paz". Imprenta Nacional, 1999.

(22) DNP. Plan de desarrollo económico y social 1990-1994 "La revolución pacífica". Imprenta Nacional, 1990.

(23) DNP. Plan nacional de desarrollo, ley de inversiones 1994-1998 "El salto social". Imprenta Nacional, 1994.

(24) Díaz Granados, L.A. El reto de la seguridad social frente a los trabajadores rurales. Más vida. N. ${ }^{\circ} 24$.

(25) El País. Gobierno liquida el Incoder y crea seis entidades para el sector rural. 7 de diciembre de 2015. 